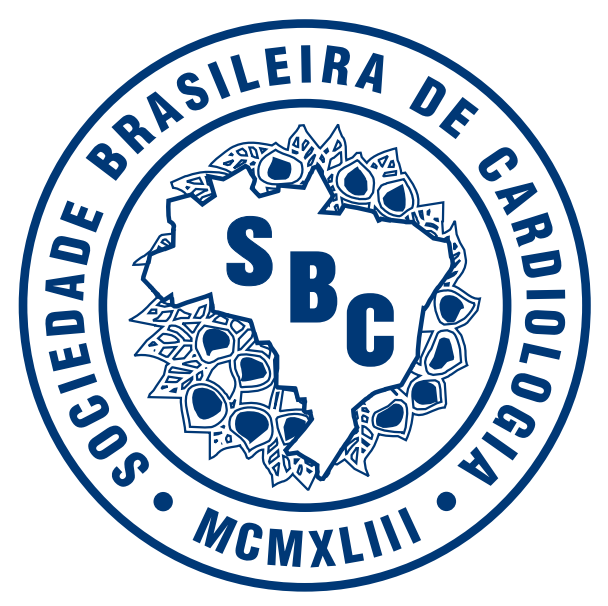

\title{
II DIRETRIZ BRASILEIRA DE CARDIOPATIA GRAVE
}

\section{EDITOR}

Oscar P. Dutra

\section{Co-Editores}

Henrique W. Besser

Humberto Tridapalli

Tiago Luiz Luz Leiria

Membros

Abrahão Afiune Neto, Antonio Felipe Simão, Antonio S. Sbissa, Enio Casagrande, Gustavo G. de Lima, Iran Castro, Iseu Gus, Ivo A. Nesralla, Nelson C. de Nonohay,

Nestor Daudt, Maria Claúdia Irigoyen, Renato A. K. Kalil

\section{Coordenador de Normatizações e Diretrizes da SBC}

\section{Jorge Ilha Guimarães}

Correspondência: Oscar Pereira Dutra - Rua Princesa Isabel, 395 - 90620-001 - Porto Alegre, RS 


\section{INTRODUÇÃo}

Inúmeras pesquisas têm demonstrado que, se, por um lado, a expectativa de vida do brasileiro cresceu nos últimos dez anos, há, por outro, um índice muito maior de doenças cardiovasculares. Verificou-se, entre essas, o incremento de situações graves, que impedem o retorno de muitos pacientes ao trabalho. Tornou-se, então, fundamental conceituar Cardiopatia Grave, para a orientação tanto do cardiologista como de colegas de outras especialidades. Com essa intenção, a Sociedade Brasileira de Cardiologia propôs esta Diretriz.

O termo Cardiopatia Grave aparece pela primeira vez na legislação brasileira com a Lei n. ${ }^{\circ} 1711$ (item III, do Artigo 178) do Estatuto dos Funcionários Civis da União, sancionada em 28 de outubro de 1952, que visava beneficiar os pacientes acometidos de moléstia profissional, acidente em serviço, tuberculose ativa, alienação mental, neoplasia maligna, cegueira, lepra, paralisia irreversível e incapacitante, cardiopatia grave e estados avançados da doença de Paget (osteíte deformante). Esta lei foi reeditada em outras ocasiões, sem modificações significativas. A partir de $1 .^{\circ}$ de janeiro de 1989 , passou a vigorar como a Lei 7713/88, incluindo, então, a síndrome de imunodeficiência adquirida (SIDA/ AIDS) e beneficiando os pacientes acometidos pelas mesmas doenças listadas na lei anterior, mesmo que tenham sido contraídas depois da aposentadoria ou reforma (Artigos $6 .^{\circ}, \mathrm{XVI}$ e $57 .^{\circ}$ ). Em 30 de dezembro de 2004, foi publicada, no Diário Oficial da União, a inclusão das hepatopatias graves, nefropatias graves, doenças causadas por radiação ionizante e a doença de Parkinson, como merecedoras do mesmo benefício.

Em 1952, uma comissão multidisciplinar de médicos enunciou o conceito de Cardiopatia Grave como doença que leva, em caráter temporário ou permanente, à redução da capacidade funcional do coração, a ponto de acarretar risco à vida ou impedir o servidor de exercer as suas atividades. A incapacitação laboral deve ser avaliada por perícia médica. Nesse procedimento, o segurado ou paciente, vítima de uma doença ou acidente de trabalho, é examinado por um profissional especializado (médico-perito), que avalia as condições de saúde e a capacidade laborativa, decidindo sobre a conveniência do afastamento ou o retorno às atividades laborativas habituais, de acordo com as normatizações contidas nos Estatutos do Funcionalismo Público Civil ou Militar dos municípios, estados e federação (Manual do Médico Perito, 1980; Perícia Médica, 1990).

0 médico-perito, diferentemente do médicocardiologista-clínico, não exerce a medicina clínica, pois não cuida de enfermos. Utiliza os conhecimentos médicos apenas para estabelecer o diagnóstico e o prognóstico clínico, para julgar a capacidade laborativa e sua imputabilidade. Assim, a atividade e o conhecimento pericial sugerem uma especialidade de cunho médicojudicial, na qual, além dos conhecimentos profundos de clínica, existe a necessidade de uma postura, raciocínio e julgamento, como fim. A adaptação do conhecimento médico às exigências das normas legais realiza-se com critérios e princípios diferentes dos que regem a apreciação dos problemas clínicos. As exigências da medicina clínica são diferentes da pericial, que se vê envolvida com a legislação, que devem sustentar o parecer pericial.

A medicina pericial exercida atualmente em muito difere da praticada há 30 ou 40 anos. Mais do que nunca, passou-se a exigir a comprovação diagnóstica por meio de uma rigorosa avaliação clínica e comprovação laboratorial (métodos complementares não-invasivos e invasivos), evitando-se as conclusões baseadas em impressões subjetivas ou alegações emanadas dos pacientes, sem o corroborativo laboratorial, tão sujeitas a erros ou interpretações enganosas.

\section{Conceituação}

O conceito de cardiopatia grave engloba tanto doenças cardíacas crônicas, como agudas. São consideradas cardiopatias graves:

a) cardiopatias agudas, habitualmente rápidas em sua evolução, que se tornam crônicas, caracterizadas por perda da capacidade física e funcional do coração;

b) as cardiopatias crônicas, quando limitam, progressivamente, a capacidade física e funcional do coração (ultrapassando os limites de eficiência dos mecanismos de compensação), não obstante o tratamento clínico e/ou cirúrgico adequado;

c) cardiopatias crônicas ou agudas que apresentam dependência total de suporte inotrópico farmacológico (como dobutamina, dopamina) ou mecânico (tipo Biopump, balão intra-aórtico);

d) cardiopatia terminal: forma de cardiopatia grave em que a expectativa de vida se encontra extremamente reduzida, geralmente não responsiva à terapia farmacológica máxima ou ao suporte hemodinâmico externo. Esses pacientes não são candidatos à terapia cirúrgica, para correção do distúrbio de base (valvopatia, cardiopatia isquêmica, cardiopatia congênita...) ou transplante cardíaco, devido à severidade do quadro clínico ou comorbidades associadas (hipertensão arterial pulmonar, disfunção renal severa, neoplasia avançada).

A limitação da capacidade física e funcional é definida, habitualmente, pela presença de uma ou mais das seguintes síndromes: insuficiência cardíaca, insuficiência coronariana, arritmias complexas, bem como hipoxemia e manifestações de baixo débito cerebral, secundárias a uma cardiopatia. A gravidade dessas síndromes será definida nas seções posteriores.

A avaliação da capacidade funcional do coração permite a distribuição dos pacientes em classes ou graus, assim descritos: 
a) GRAU I: pacientes portadores de doença cardíaca sem limitação da atividade física. A atividade física normal não provoca sintomas de fadiga acentuada, nem palpitações, nem dispnéias, nem angina de peito, nem sinais e sintomas de baixo fluxo cerebral;

b) GRAU II: pacientes portadores de doenças cardíacas com leve limitação da atividade física. Estes pacientes sentem-se bem em repouso, porém os grandes esforços provocam fadiga, dispnéia, palpitações ou angina de peito;

c) GRAU III: pacientes portadores de doença cardíaca com nítida limitação da atividade física. Estes pacientes sentem-se bem em repouso, embora acusem fadiga, dispnéia, palpitações ou angina de peito, quando efetuam pequenos esforços;

d) GRAU IV: pacientes portadores de doença cardíaca que os impossibilita de qualquer atividade física. Estes pacientes, mesmo em repouso, apresentam dispnéia, palpitações, fadiga ou angina de peito.

Os meios de diagnósticos a serem empregados na avaliação da capacidade funcional do coração são: história clínica, com dados evolutivos da doença; exame clínico; eletrocardiograma, em repouso; eletrocardiografia dinâmica (Holter); teste ergométrico; teste de caminhada de 6 minutos; ergoespirometria (VO2 pico < 14ml/kg/min); questionário de qualidade de vida SF-36; ecocardiograma, em repouso; ecocardiograma associado a esforço ou procedimentos farmacológicos; estudo radiológico do tórax, objetivando o coração, vasos e campos pulmonares, usando um mínimo de duas incidências; cintilografia miocárdica, associada ao teste ergométrico (tálio, MIBI, tecnécio); cintilografia miocárdica associada a dipiridamol e outros fármacos; cinecoronarioventriculografia; angiotomografia computadorizada, tomografia coronariana computadorizada, angio-ressonância magnética e ressonância magnética cardíaca.

Nos portadores de doença cardíaca não identificável com os meios de diagnósticos citados, podem ser utilizados outros exames e métodos complementares, como biópsia miocárdica.

Os achados fortuitos em exames complementares especializados não são, por si só, suficientes para o enquadramento legal de cardiopatia grave.

O quadro clínico bem como os recursos complementares, com os sinais e sintomas que permitem estabelecer o diagnóstico de cardiopatia grave, estão relacionados às seguintes cardiopatias: cardiopatia isquêmica, cardiopatia hipertensiva, miocardiopatias, valvopatias, cardiopatias congênitas, arritmias, pericardiopatias, aortopatias e cor pulmonale crônico.

Em algumas condições, um determinado item pode, isoladamente, configurar cardiopatia grave (por exemplo, fração de ejeção $<0,35$ ), porém, na grande maioria dos casos, é necessária uma avaliação conjunta dos diversos dados do exame clínico e dos achados complementares para melhor conceituá-la.
Nas seções a seguir, serão definidos os tópicos importantes a serem valorizados na definição de gravidade das diferentes cardiopatias.

\section{1 - CARdiopatia IsQUÊMICA}

\section{Forma aguda (infarto agudo do miocárdio/angina instável)}

Síndromes coronarianas agudas sem supradesnivelamento de segmento ST com $^{1,2}$ - Sinais de baixo débito cardíaco; sinais de insuficiência cardíaca aguda; presença de arritmia ventricular maligna; sinais de disfunção ventricular mecânica; os tópicos acima, em paciente já revascularizado, sem condições de tratamento cirúrgico ou percutâneo.

Síndromes coronarianas agudas com supradesnivelamento do segmento ST ou BRE novo ${ }^{3,4}$ - Choque cardiogênico (Killip IV); sinais de insuficiência cardíaca aguda (Killip II-III); presença de arritmia ventricular maligna; complicação mecânica do IAM (ruptura de parede livre, CIV, disfunção de músculo papilar); IAM anterior extenso (V1-V6 DI aVL); presença de BAV II, grau Mobiz II, BAVT, ou distúrbio de condução intraventricular; infarto perioperatório de CRM; quadro clínico de infarto agudo do miocárdio, em paciente já com infarto prévio de grande extensão, ou com IC já estabelecida.

\section{Forma crônica (angina estável) ${ }^{5-17}$}

Quadro clínico de forma crônica - Angina classes III e IV da CCS (Canadian Cardiovascular Society), apesar da terapêutica máxima adequadamente usada; manifestações clínicas de insuficiência cardíaca, associada à isquemia aguda nas formas crônicas, a presença de disfunção ventricular progressiva; arritmias graves associadas ao quadro anginoso, principalmente do tipo ventricular (salvas de extra-sístoles, taquicardia ventricular não sustentada ou sustentada (devem-se associar dados do ECG e Holter).

Eletrocardiograma (repouso) - Zona elétrica inativa (localização e magnitude), alterações permanentes e significativas na repolarização ventricular; alterações isquêmicas de ST-T(tipo segmento ST permanentemente elevado, configurando a possibilidade de aneurisma ventricular); distúrbios da condução atrioventricular e intraventricular. (QRS maior que 120 ms); hipertrofia ventricular esquerda, de grande magnitude; fibrilação atrial crônica; arritmias ventriculares complexas (associar com dados do Holter).

Radiografia do tórax - Cardiomegalia com índice cardiotorácico superior 0,5 , o qual possui valor prognóstico definido; congestão venocapilar pulmonar; derrame pleural bilateral ou unilateral importante.

Teste ergométrico - Limitação da capacidade funcional ( $<5 \mathrm{MET}$ ); angina em carga baixa ( $<5 \mathrm{MET}$ ); infradesnível 
do segmento ST: precoce (carga baixa), acentuada ( $>3 \mathrm{~mm}$ ), morfologia horizontal ou descendente, múltiplas derivações, duração prolongada ( $>6$ min no período de recuperação). Supradesnível de ST, sobretudo em área não relacionada a infarto prévio; comportamento anormal da pressão arterial diastólica: queda de PAS $>30 \mathrm{mmHg}$; insuficiência cronotrópica (elevação inadequada da freqüência cardíaca, descartado o uso de drogas que possam alterar o cronotropismo); sinais de disfunção ventricular esquerda associada ao esforço; arritmias ventriculares complexas, durante ou pós-esforço.

Cintilografia miocárdica associada a teste ergométrico (tálio, MIBI, tecnécio) - Defeitos de perfusão múltiplos ou áreas extensas (áreas hipocaptantes definitivas ou transitórias); dilatação da cavidade ventricular esquerda ao esforço; hipercaptação pulmonar; fração de ejeção (FE) em repouso ou esforço $\leq(40 \%)$ (valor específico para o método); comportamento anormal da FE ao exercício (variação da $\mathrm{FE}<5 \%$ ); mobilidade parietal regional ou global anormal.

Cintilografia miocárdica associada a dipiridamol e outros fármacos - Interpretação semelhante à definida para a cintilografia com teste ergométrico.

Ecocardiograma (em repouso) - Fração de ejeção $<0,40$ (valor específico para o método). Alterações segmentares de grande magnitude ou vários segmentos que modificam a contratilidade ventricular, levando à redução da fração ventricular; dilatação das câmaras esquerdas, especialmente se associadas à hipertrofia ventricular esquerda; complicações associadas: disfunção dos músculos papilares, insuficiência mitral, comunicação interventricular, pseudo-aneurismas, aneurismas, trombos intracavitários; complicações associadas ao esforço ou aos procedimentos farmacológicos; aparecimento de alterações da contratilidade segmentar inexistentes no ecocardiograma em repouso. Anormalidades em dois segmentos da parede ventricular em repouso ou induzidas com doses baixas de dobutamina; resposta inotrópica inadequada ao uso de drogas cardioestimulantes; acentuação das alterações de contratilidade preexistentes; comportamento anormal da FE ao exercício (variação da $\mathrm{FE}<5 \%$ ).

Eletrocardiografia dinâmica (Holter) - Alterações isquêmicas dinâmicas (ST-T--t), associadas ou não à dor anginosa, com ou sem sintomas de disfunção ventricular esquerda; isquemia miocárdica silenciosa; arritmias ventriculares complexas, transitórias ou não; fibrilação atrial e flutter atrial associados à isquemia; distúrbios de condução atrioventricular e intraventricular relacionados à isquemia - bloqueios de ramos induzidos pelo esforço físico.

Cinecoronarioventriculografia - Lesão de tronco de coronária esquerda $>50 \%$; lesões em três vasos, moderadas a importantes ( $>70 \%$ em $1 / 3$ proximal ou médio) e, eventualmente, do leito distal, dependendo da massa miocárdica envolvida; lesões em 1 ou 2 vasos de $>70 \%$, com grande massa miocárdica em risco; lesões ateromatosas extensas e difusas, sem viabilidade de correção cirúrgica ou por intervenção percutânea; fração de ejeção $<0,40$; hipertrofia e dilatação ventricular esquerdas; áreas extensas de acinesia, hipocinesia e discinesia; aneurisma de ventrículo esquerdo; complicações mecânicas: insuficiência mitral, comunicação interventricular.

Fatores de risco e condições associadas - Idade $>70$ anos, hipertensão, diabetes, hipercolesterolemia familiar; vasculopatia aterosclerótica importante em outros territórios, como carótidas, membros inferiores, renais, cerebrais.

Pós-infarto do miocárdio - Cintilografia com Tálio / PET com FDG /RNM para pesquisa de viabilidade e demarcação de necrose, pois a extensão de necrose é marca de gravidade; disfunção ventricular esquerda (áreas de acinesia, hipocinesia e discinesia);

isquemia à distância (em outra área que não a do infarto); arritmias ventriculares complexas; idade avançada.(> 70 anos); comorbidades associadas, como diabetes mellitus; doença pulmonar obstrutiva severa, neoplasias, doença arterial periférica, comprometendo vários leitos arteriais.

Quando o tratamento adequado - clínico, intervencionista ou cirúrgico - melhorar ou abolir as alterações acima descritas, o conceito de gravidade deve ser reconsiderado e reavaliado.

No entanto o critério de gravidade mantém-se se a fração de ejeção detectada permanecer com valores inferiores a $40 \%$, ou a área de necrose quantificada por um dos métodos acima apresentar valor $>20 \%$, ou ainda houver detecção de arritmias malignas.

\section{2 - Cardiopatia Hipertensiva ${ }^{18-24}$}

A definição de cardiopatia grave na doença hipertensiva não depende exclusivamente dos níveis tensionais, mas da concomitância de lesões em órgãos-alvos: rins, coração, cérebro, retina e artérias periféricas.

Se a pressão diastólica for menor do que $110 \mathrm{mmHg}$ e acompanhada de danos a órgão(s)-alvo, é definida como cifra baixa complicada. Se a pressão diastólica for $>110 \mathrm{mmHg}$ e acompanhada de dano a órgão(s)-alvo, é definida como cifra alta complicada. Os órgãos-alvo que podem ser comprometidos por uma cifra baixa durante longo tempo ou por cifras altas durante curto tempo são coração, cérebro, rins, retina e artérias periféricas.

O comprometimento do coração na hipertensão arterial identifica a cardiopatia hipertensiva. Quando isto ocorre, freqüentemente os demais órgãos-alvo também podem estar comprometidos. De outra parte, em alguns casos, um ou mais dos órgãos-alvo podem estar envolvidos, sem que o coração o esteja. Nesses casos, não se trata de cardiopatia hipertensiva, mas de hipertensão arterial complicada.

Na cardiopatia hipertensiva, a gravidade é caracterizada 
pela presença das seguintes condições: hipertrofia ventricular esquerda detectada pelo ECG com alterações da repolarização ventricular ou ecocardiograma com massa ventricular esquerda acima de $163 \mathrm{~g} / \mathrm{m}$ em homens e $121 \mathrm{~g} / \mathrm{m}$ em mulheres que não regride com o tratamento, disfunção ventricular esquerda sistólica, com fração de ejeção $<0,40 \%$, arritmias supraventriculares e ventriculares complexas relacionadas à hipertensão arterial e cardiopatia isquêmica grave associada.

A cardiopatia hipertensiva é agravada, ainda, pelo comprometimento de outros órgãos-alvo, como discriminado a seguir: em relação ao cérebro: isquemia cerebral transitória, acidente vascular cerebral isquêmico ou hemorrágico.

Em relação aos rins é caracterizado por: creatinina $>3,0 \mathrm{mg} / \mathrm{dl}$, ou DCE abaixo de $30 \mathrm{ml} / \mathrm{min}$; presença de albuminúria e/ou sinais de insuficiência renal crônica (redução do tamanho dos rins, disfunção plaquetária, anemia crônica, distúrbio do equilíbrio ácido básico, hiperazotemia).

Em relação a artérias periféricas é caracterizado por: aneurisma e/ou dissecção da aorta, trombose arterial periférica, estenose de carótida $>70 \%$ assintomática e $>50 \%$ com sintomas.

Em relação à retina é caracterizado por: hemorragias, exsudato e papiledema, especialmente quando não regridem com tratamento adequado.

\section{3 - Miocardiopatias $25-29$}

Miocardiopatias hipertróficas - Presença de um ou mais fatores abaixo: paciente sintomático, especialmente com história de síncope, angina, insuficiência cardíaca e embolia sistêmica; diagnóstico na infância (baixa idade); hipertrofia moderada ou severa, com alterações isquêmicas de ST-T; cardiomegalia; disfunção ventricular esquerda sistólica, com fração de ejeção abaixo de 40\%; fibrilação atrial; síndrome de Wolff-Parkinson-White associada; arritmias ventriculares complexas; regurgitação mitral importante; doença arterial coronariana grave associada; forma obstrutiva com gradiente de via de saída $>50 \mathrm{mmHg}$; perfil citogenético de alto risco.

Miocardiopatias dilatadas (primárias ou secundárias) - Presença de um ou mais fatores abaixo: história de fenômenos tromboembólicos sistêmicos; cardiomegalia importante; ritmo de galope (B3); insuficiência cardíaca classe funcional III e IV; fração de ejeção <0,40; fibrilação atrial; arritmias ventriculares complexas; distúrbios da condução intraventricular, com complexos QRS > 120mms ou presença de assincronia ventricular demonstrada por ecocardiograma, com Doppler tissular.

Miocardiopatia restritiva (endomiocardiofibrose, fibroelastose, miocardiopatias infiltrativas-amiloidose) - Presença de um ou mais fatores abaixo: história de fenômenos tromboembólicos; cardiomegalia acentuada; insuficiência cardíaca classe funcional III e IV; envolvimento do ventrículo direito; fibrose miocárdica acentuada; regurgitação mitral e/ou de tricúspide importante.

Cardiopatia chagásica crônica - História de síncope; fenômenos tromboembólicos; cardiomegalia acentuada; insuficiência cardíaca classe funcional III e IV; fibrilação atrial; arritmias ventriculares complexas; bloqueio bi ou trifascicular sintomático; bloqueio atrioventricular total.

\section{4 - VALVOPATIAS $30-32$}

\section{Insuficiência mitral}

Quadro clínico - insuficiência cardíaca classe funcional III e IV; frêmito sistólico palpável na região da ponta; primeira bulha inaudível ou acentuadamente hipofonética no foco mitral; sopro holossistólico no foco mitral, de intensidade $>3 / 6$, com irradiação em faixa ou círculo; segunda bulha hiperfonética no foco pulmonar; desdobramento amplo e constante da segunda bulha no foco pulmonar; insuficiência mitral aguda associada ou não a processo isquêmico.

Eletrocardiograma - Sinais progressivos de sobrecarga atrial e ventricular esquerdas, com alterações da repolarização ventricular; fibrilação atrial.

Radiografia de tórax - Aumento acentuado da área cardíaca, como predominância das cavidades esquerdas; sinais de congestão venocapilar pulmonar; sinais de hipertensão pulmonar.

Ecocardiograma - Presença de jato regurgitante de grande magnitude; comprometimento progressivo da função ventricular sistólica; aumento significativo do diâmetro sistólico do ventrículo esquerdo; inversão do fluxo sistólico em veia pulmonar; sinais de hipertensão pulmonar.

Hemodinâmica e angiografia - Onda " $v$ " com valor $>3$ vezes, em relação à média do capilar pulmonar; opacificação do átrio esquerdo igual ou maior que a do ventrículo esquerdo: graus III e IV da classificação de Sellers; fração de regurgitação $>60 \%$ (FR - volume de regurgitação/volume sistólico total).

\section{Estenose mitral}

Quadro clínico - História de comissurotomia mitral prévia; fenômenos tromboembólicos; insuficiência cardíaca classe funcional III e IV; episódios de edema pulmonar agudo; escarros hemópticos; fibrilação atrial; estalido precoce de abertura da valva mitral; impulsão sistólica de ventrículo direito; segunda bulha hiperfonética no foco pulmonar; sinais de insuficiência tricúspide.

Eletrocardiograma - Fibrilação atrial; sinais de sobrecarga de câmaras direitas.

Estudo radiográfico - Inversão do padrão vascular pulmonar; sinais de hipertensão venocapilar pulmonar; sinais de hipertensão arteriolar pulmonar.

Ecocardiograma - Área valvar $<1,0 \mathrm{~cm}^{2}$; tempo de $1 / 2$ 
pressão $>200 \mathrm{~ms}$; gradiente transvalvar mitral médio $>15 \mathrm{mmHg}$; sinais de hipertensão pulmonar (pressão sistólica da artéria pulmonar $>50 \mathrm{mmHg}$ ); presença de trombo no átrio esquerdo.

Hemodinâmica - Área valvar $<1,0 \mathrm{~cm}^{2}$; gradiente diastólico mitral médio $>15 \mathrm{mmHg}$; pressão média de capilar pulmonar ou de átrio esquerdo $>20 \mathrm{mmHg}$; pressão sistólica da artéria pulmonar $>50 \mathrm{mmHg}$.

\section{Insuficiência aórtica}

Quadro clínico - Insuficiência cardíaca classe funcional III e IV; manifestações de baixo débito cerebral (tontura, lipotímia, síncope); síndrome de Marfan associada; presença de galope ventricular (B3); sopro de Austin-Flint na ponta; íctus hipercinético, deslocado externamente; pressão diastólica próxima a zero; queda progressiva da pressão arterial sistólica; presença de aneurisma do seio de Valsalva.

Eletrocardiograma - Sinais de sobrecarga ventricular esquerda com onda T negativa em precordiais esquerdas; sinais de sobrecarga atrial esquerda; fibrilação atrial.

Estudo radiográfico - Aumento importante da área cardíaca, com franco predomínio de ventrículo esquerdo (aspecto em "bota"); dilatação da aorta ascendente, da croça e do segmento descendente; dilatação do átrio esquerdo.

Ecocardiograma - Jato regurgitante Ao/VE largo e extenso; fluxo reverso holodiastólico da aorta descendente; abertura valvar mitral somente com a sístole atrial; piora progressiva dos parâmetros da função sistólica ventricular esquerda; queda da fração de ejeção ao ecocardiograma de esforço, abaixo de 40\%.

Cintilografia miocárdica - Comportamento anormal da fração de ejeção, abaixo de 40\%.

Hemodinâmica e angiografia - Baixa pressão diastólica da aorta, tendendo à equalização das pressões diastólicas aorto-ventriculares; pressão diastólica final do ventrículo esquerdo (Pd2 VE) elevada (>20 mmHg); opacificação igual ou mais densa do ventrículo esquerdo em comparação com a aorta, durante aortografia (Graus III e IV de Sellers); fração de regurgitação > 60\%.

\section{Estenose aórtica}

Quadro clínico - Sintomas de baixo débito cerebral (tontura, lipotimia, síncope); angina de peito; presença de terceira bulha; insuficiência cardíaca; pressão arterial diferencial reduzida; pico tardio de intensidade máxima do sopro; desdobramento paradoxal da segunda bulha; fibrilação atrial.

Eletrocardiograma - Sinais de sobrecarga ventricular esquerda importante, com infradesnivelamento de ST e onda $\mathrm{T}$ negativa em precordiais esquerdas; sobrecarga atrial esquerda; fibrilação atrial; arritmias ventriculares complexas; bloqueio atrioventricular total.
Ecocardiograma - Área valvar $<0,75 \mathrm{~cm}^{2}$; gradiente médio de pressão transvalvar aórtica > $50 \mathrm{mmHg}$; gradiente máximo > $70 \mathrm{mmHg}$; sinais de hipocinesia ventricular esquerda.

Hemodinâmica - Área valvar $<0,75 \mathrm{~cm}^{2}$; hipocinesia ventricular esquerda; coronariopatia associada.

Situação pós-cirúrgica - Dadas as condições hemodinâmicas que uma troca proporciona, é de consenso que a obstrução permanece, embora com um gradiente menor, mantendo, portanto, a condição fisiopatológica. A permanência da definição de cardiopatia grave dependerá do gesto profissional.

\section{Prolapso valvar mitral}

História familiar de morte súbita; história de síncope; fenômenos tromboembólicos; síndrome de Marfan associada; arritmias ventriculares complexas; fibrilação atrial; disfunção ventricular esquerda; regurgitação mitral importante; prolapso valvar tricúspide associado; cardiomegalia (aumento de câmaras esquerdas); rotura de cordoalhas tendíneas.

\section{Pacientes portadores de prótese cardíaca}

Presença de hemólise com necessidade de hemoterapia; sinais de disfunção protética aguda ou crônica; história de endocardite pregressa ou atual; presença de gradiente não funcional, acarretando disfunção/dilatação ventricular.

\section{5 - Pericardites $33-35$}

As pericardiopatias podem ser consideradas graves, principalmente nas situações de extrema restrição do enchimento ventricular, por inflamação crônica. Devem estar presentes também achados clínicos associados ao quadro de restrição ventricular, como congestão circulatória periférica, pulso paradoxal, turgência jugular, refluxo hepato-jugular, hepatomegalia.

As grandes restrições pericárdicas devem-se às seguintes patologias:

a) por tuberculose - forma decorrente da infecção pericárdica pelo Mycobacterium tuberculosis. Em muitos adultos, a pericardite por Tbc representa reativação da doença. A pericardite por Tbc pode ser dividida em estágios: 1) seco; 2) efusivo; 3) absortivo; 4) constritivo (como seqüela tardia);

b) por radiação - pericardite tardia pode ocorrer de forma abrupta - ou como derrame pericárdico de instalação gradual, ou como forma constritiva. Na lesão por radiação, pode ocorrer doença também das coronárias e do miocárdio.

\section{6 - Cor Pulmonale Crônico 36,37}

Quadro clínico - Manifestações de hipóxia cerebral e periférica (dedos em baqueta de tambor); insuficiência 
cardíaca direita; angina de peito classe III a IV da NYHA. Crises sincopais; hiperfonese clangorosa da segunda bulha no foco pulmonar; galope ventricular direito (B3). Gasometria arterial com PO2 <60mmHg; PCO2 >50mmHg.

Eletrocardiograma - Sinais de sobrecarga importante de câmaras direitas.

Ecocardiografia - Hipertrofia ventricular direita com disfunção diastólica e/ou sistólica; grande dilatação do átrio direito; pressão sistólica em artéria pulmonar, calculada com base nas pressões do VD e AD, $>60 \mathrm{mmHg}$; insuficiência tricúspide importante; inversão do fluxo venoso na sístole atrial.

Estudo hemodinâmico - Dilatação do tronco da artéria pulmonar. Dilatação do ventrículo direito; dilatação do átrio direito; pressão na artéria pulmonar $>60 \mathrm{mmHg}$; pressão no átrio direito > $15 \mathrm{mmHg}$; insuficiência pulmonar; insuficiência tricúspide.

\section{7 - Cardiopatias Congênitas ${ }^{38}$}

Caracterizam-se como graves as cardiopatias congênitas que apresentam:

Do ponto de vista clínico: crises hipoxêmicas; insuficiência cardíaca (classe III e IV); hemoptises, pela presença de circulação colateral brônquica; arritmias de difícil controle e potencialmente malignas.

Do ponto de vista anatômico - Doença arterial pulmonar; necrose miocárdica por doença coronariana ou origem anômala das artérias coronarianas; drenagem anômala total infracardíaca ou com obstruções severas da conexão das veias pulmonares com as sistêmicas; hipotrofia ventricular direita; agenesias valvares (pulmonar e aórtica); hipoplasia ou atresia de valvas pulmonares, aórtica e mitral; hipoplasia ou atresia do coração esquerdo; estenose mitral; transposição das grandes artérias com hiper-resistência pulmonar ou ausência de comunicações; ventrículos únicos com atresias valvares; ectopias cardíacas com alterações múltiplas; cardiopatias complexas.

Do ponto de vista anátomo-funcional - Sobrecargas diastólicas ventriculares associadas à hipocontratilidade ventricular acentuada, com manifestações clínicas; sobrecargas sistólicas ventriculares com hipertrofia importante e desproporcionada ou com miocardioesclerose e manifestações clínicas; cardiopatias hipertróficas acentuadas, com manifestações clínicas; nas formas crônicas com defeitos corrigidos, a presença de ICE, ICD ou ICC e seus achados clínicos - enteropatia perdedora de proteínas, ascite refratária, cirrose cardíaca.

\section{8 - Doenças Da Aorta ${ }^{40-45}$}

As doenças da aorta, principalmente em sua porção torácica, são patologias com morbi-mortalidade elevada. Tanto o tratamento clínico como cirúrgico ainda estão relacionados a elevadas taxas de mortalidade, tornando esse grupo de patologias alvo de extrema importância no tópico das cardiopatias graves.

\section{Aneurisma de aorta torácica}

Considera-se a indicação de cirurgia cardíaca quando o diâmetro maior de 5,5 cm na porção ascendente da aorta; com diâmetro maior de $6 \mathrm{~cm}$ na porção descendente da aorta; com diâmetro entre 6 e $7 \mathrm{~cm}$ nos pacientes de alto risco cirúrgico; com qualquer diâmetro associado a sintomas por compressão de estruturas vizinhas (rouquidão, dor e dispnéia); com diâmetro maior de 5 $\mathrm{cm}$ nos portadores de síndrome de Marfan ou válvula aórtica bicúspede; com diâmetro maior de $4 \mathrm{~cm}$, em pacientes com válvula aórtica bicúspede, que necessitem de correção cirúrgica da valvopatia. A indicaçãocirúrgica está indicada também nos aneurismas de aorta associado à rápida progressão, história familiar de dissecção ou estados hipermetabolismo (gestação).

\section{Dissecção de aorta}

Dissecção aguda de aorta (menos de quinze dias entre os sintomas e o diagnóstico), envolvendo porção ascendente (Tipo A - Stanford), com ou sem envolvimento da válvula aórtica; dissecção aguda de aorta (Tipo B - Stanford), associada a comprometimento de órgão alvo, ruptura ou iminência de ruptura (formação sacular), extensão retrógrada e nos portadores de síndrome de Marfan; dissecção não aguda da aorta associada com envolvimento de órgão alvo, sintomas recorrentes, progressão retrógrada ou anterógrada da lesão intimal, já pré-estabelecida.

\section{Hematoma de aorta}

Os hematomas intramurais de aorta apresentam prognóstico semelhante aos da dissecção clássica de aorta; aqueles localizados na porção proximal da aorta são de pior prognóstico.

\section{Conclusão}

É correta a afirmativa de Besser ${ }^{46}$ de que "É preciso não confundir gravidade de uma cardiopatia com Cardiopatia Grave, uma entidade médico-pericial".

Essencialmente, a classificação de uma Cardiopatia Grave não é baseada em dados que caracterizam uma entidade clínica, e sim, nos aspectos de gravidade das cardiopatias, colocados em perspectiva com a capacidade de exercer as funções laborativas e suas relações como prognóstico de longo prazo e a sobrevivência do indivíduo.

Verifica-se uma dificuldade ainda maior na extensão do benefício aos inativos (aposentados), já que estes indivíduos não mais exercem a atividade laborativa (esforço físico), um dos fatores considerados importantes no julgamento pericial de incapacitação. 
Embora os procedimentos intervencionistas e cirúrgicos sejam considerados na medicina pericial apenas parte da estratégia terapêutica aplicada aos doentes e, obviamente, não sejam considerados uma doença propriamente dita, sabemos que a cada intervenção corresponde uma enfermidade cardiovascular importante subjacente, que deverá ser avaliada em relação à ação deletéria e às deficiências funcionais que se possam imputar sobre a capacitação laboral do doente, como em todas as cardiopatias. Sabemos, também, que, num grande número de pacientes, a cirurgia ou o procedimento intervencionista alteram efetivamente a história natural da doença para melhor, modificando radicalmente a evolução de muitas doenças e, conseqüentemente, a categoria da gravidade da cardiopatia, pelo menos no

\section{REFERÊNCIAS}

1. Diretriz da Sociedade Brasileira de Cardiologia sobre Angina Instável e Infarto do Miocárdio sem Supradesnível do Segmento ST. Arq Bras Cardiol. 2001; 77(supl II):1-38.

2. Braunwald E, Antman EM, Beasley JW,et al. ACC/AHA 2002 Guideline update for the management of patients with unstable angina and non-ST - segment elevation myocardial infarction - summary article: A report of the American College of Cardiology/American Heart Association Task Force on practice guidelines (Committee on the Management of patients with Unstable Angina). J Am Coll Cardiol. 2002;40:1366-74

3. SBC - III Diretriz sobre Tratamento do Infarto Agudo do Miocárdio. Arq Bras Cardiol. 2004;83(supl IV): 1-86.

4. Antman EM, Anbe DT, Armstrong PW, et al. Task Force on practice guidelines (writing committee to revise the 1999 guidelines for the management of patients with acute myocaqrdial infarction). ACC/ AHA guidelines for the management of patients with ST-elevation myocardial infarction - executive summary: A report of the American College of Cardiology/American Heart Association. J Am Coll Cardiol. 2004;44:671-719.

5. SBC - Diretrizes de Doença Coronariana Crônica - Angina Estável. Arq Bras Cardiol. 2004;83(supl II):1-43.

6. Sociedade Brasileira de Cardiologia. Diretrizes de Cirurgia "Revascularização Miocárdica, Valvopatias e Doenças da Aorta". Arq Bras Cardiol. 2004;82(Supl V):1-30.

7. Stenestrand U, Tabrizi F, Lindback J, Englund A, Rosenqvist $\mathrm{M}$, Wallentin L. Comorbidity and myocardial dysfunction are the main explanations for the higher 1 -year mortality in acute myocardial infarction with left bundle-branch block. Circulation. 2004;110(14): 1896-902.

8. Shlipak MG, Go AS, Frederick PD, Malmgren J, Barron HV, Canto JG. Treatment and outcomes of left bundle-branch block patients with myocardial infarction who present without chest pain. National Registry of Myocardial Infarction 2 Investigators. J Am Coll Cardiol. 2000;36:706-12.

9. Grady TA, Chiu AC, Snader CE, Marwick TH, Thomas JD, Pashkow FJ, et al. Prognostic significance of exercise-induced left bundle-branch block. JAMA. 1998;279(2):153-6.

10. Ernst ER, Shub C, Bailey KR, Brown LR, Redfield MM. Radiographic measurements of cardiac size as predictors of outcome in patients with dilated cardiomyopathy. J Card Fail. 2001;7(1):13-20.

11. II Diretrizes da Sociedade Brasileira de Cardiologia sobre Teste Ergométrico. Arq Bras Cardiol. 2002;78(supl II):1-18.

12. Udelson JE, Dilsizian V, Bonow RO, et al. Nuclear Cardiology. In: Zipes DP, Libby P, Bonow RO, Braunwald E, eds. Heart Disease: A Textbook momento da avaliação. Este é o conceito dinâmico de "reversibilidade" da evolução das cardiopatias, que deixam de configurar uma condição de Cardiopatia Grave observada anteriormente. De qualquer forma, nunca devemos achar, de antemão, que pacientes submetidos a quaisquer das intervenções mencionadas têm a condição médico-pericial de Cardiopatia Grave, como erroneamente interpretado por muitos. Considera-se um servidor (ativo ou inativo) como portador de Cardiopatia Grave, quando existir uma doença cardíaca que acarrete o total e definitivo impedimento das condições laborativas, existindo, implicitamente, uma expectativa de vida reduzida ou diminuída, baseando-se o avaliador na documentação e no diagnóstico da cardiopatia.

of Cardiovascular Medicine, $7^{\text {th }}$ edition. Philadelphia: Elsevier-Science, 2004:287-331.

13. I Diretriz da Sociedade Brasileira de Cardiologia Sobre Cardiologia Nuclear. Arq Bras Cardiol. 2002;78(supl III):1-42.

14. Diretriz para Indicações e Utilização da Ecocardiografia na Prática Clínica. Arq Bras Cardiol. 2004; 82(supl II):12-34.

15. Carney RM, Blumenthal JA, Freedland KE, et al. Low heart rate variability and the effect of depression on post-myocardial infarction mortality. Arch Intern Med. 2005;165(13):1486-91.

16. Diretriz para Realização de Exames Diagnósticos e Terapêuticos em Hemodinâmica. Arq Bras Cardiol. 2004;82(supl I): 1-6.

17. Mauss O, Klingenheben T, Ptaszynski P, Hohnloser SH. Bedside risk stratification after acute myocardial infarction: prospective evaluation of the use of heart rate and left ventricular function. J Electrocardiol. 2005;38(2):106-12.

18. IV Diretrizes Brasileiras de Hipertensão Arterial. Arq Bras Cardiol. 2004;82(supl IV):1-22.

19. Guidelines Committee. 2003 European Society of HypertensionEuropean Society of Cardiology Guidelines for the management of arterial hypertension. J Hypertens. 2003;21:1011-53.

20. The Seventh Report of the Joint National Committee on Prevention, Detection, Evaluation, and Treatment of High Blood Pressure: the JNC 7 report. JAMA. 2003;289(19):2560-72.

21. Vaur L, Gueret P, Lievre M, Chabaud S, Passa P and DIABHYCAR Study Group (type 2 DIABetes, Hypertension, CARdiovascular Events and Ramipril) study. Development of congestive heart failure in type 2 diabetic patients with microalbuminuria or proteinuria. Diabetes Care. 2003;26:855-60.

22. Borch-Johnsen K, Feldt-Rasmussen B, Strandgaard S, Schroll M, Jensen JS. Urinary albumin excretion. An independent predictor of ischemic heart disease. Arterioscler Thromb Vasc Biol. 1999;19:1992-7.

23. Anavekar NS, McMurray JJV, Velazquez EJ, Solomon SD, Kober $\mathrm{L}$, Rouleau J-L, et al. Relation between renal dysfunction and cardiovascular outcomes after myocardial infarction. N Engl J Med. 2004;351:1285-95.

24. Goss LZ, Rosa RM, O'Brien WM, Ayers CR, Wood JE.. Predicting death from renal failure in primary hypertension. Arch Intern Med. $1969 ; 124(2): 160-4$

25. I Diretriz Latino-Americana para Avaliação e Conduta na Insuficiência Cardíaca Descompensada. Arq Bras Cardiol. 2005; 85(Supl III):1-48.

26. Revisão das II Diretrizes da Sociedade Brasileira de Cardiologia para o Diagnóstico e Tratamento da Insuficiência Cardíaca. Arq Bras Cardiol. 


\section{2; Vol 79(Supl IV):1-30}

27. Swedberg (Chairperson) K, Cleland J, Dargie H, Drexler H, Follath F, Komajda AM, et al. Chronic Heart Failure (Diagnosis and Treatment of)- Executive Summary and Full Text. Eur Heart J. 2005; 26:11151140.

28. Maron BJ, McKenna (Chairpersons) WJ, Danielson GK, Kappenberger LJ, Kuhn HJ, Seidman CE, et al. Wigle Hypertrophic Cardiomyopathy (Clinical Expert Consensus Document on). Eur Heart J. 2003;24(21):1965-91.

29. Diagnosis and Management of Chronic Heart Failure in the Adult: ACC/ AHA 2005 Guideline Update Circulation. 2005;112: (electronic)

30. Valvular Heart Disease: ACC/AHA Practice Guidelines for the Management of Patients with ST-elevation myocardial infarction. J Am Coll Cardiol. 1998; 32: 1486-588.

31. Recommendations on the management of the asymptomatic patient with valvular heart disease. Eur Heart J. 2002:23:1253-66.

32. Sociedade Brasileira de Cardiologia. Diretrizes de Cirurgia "Revascularização Miocárdica, Valvopatias e Doenças da Aorta". Arq Bras Cardiol. 2004; 82(Supl V):1-30.

33. Ortbals DW, Avioli LV. Tuberculous pericarditis. Arch Intern Med. 1979;139:231-4.

34. Fajardo LF, Stewart JR. Pathogenesis of radiation-induced myocardial fibrosis. Lab Invest. 1973; 29:244.

35. The Task Force on the Diagnosis and Management of Pericardial Diseases of the European Society of Cardiology. Eur Heart J. 2004;25:587-610.

36. I Consenso Brasileiro de Doença Pulmonar Obstrutiva Crônica. Jornal de Pneumologia. 2000; Vol 26(Supl 1)
37. Romano PM, Peterson S. The management of cor pulmonale. Heart Dis. 2000;2:431-7.

38. Task force of the ESC. Grown-Up Congenital Heart Disease. Eur Heart J. 2003; 24(11):1035-84.

39. DeSanctis R, Doroghazi W, Austen RM, Buckley MJ. Aortic dissection. N Engl J Med. 1987;317:1060-7.

40. Hagan PG, Nienaber CA, Isselbacher EM, Bruckman D, Karavite DJ, Russman PL, et al. The International Registry of Acute Aortic Dissection (IRAD): New Insights Into An Old Disease. JAMA. 2000;283:897-903.

41. Nienaber CA, Eagle KA. Aortic dissection: new frontiers in diagnosis and management, part I: from etiology to diagnostic strategies. Circulation. 2003;108:628-35.

42. Nienaber CA, von Kodolitsch $Y$, Petersen B, Loose R, Helmchen $\mathrm{U}$, Haverich A, et al. Intramural hemorrhage of the thoracic aorta: diagnostic and therapeutic implications. Circulation. 1995;92:146572

43. Kaji S, Akasaka T, Horibata Y, Nishigami K, Shono H, Katayama M, et al. Long-term prognosis of patients with type $A$ aortic intramural hematoma. Circulation. 2002;106(Suppl I):I-248-I-252.

44. Mehta RH, O'Gara PT, Bossone E, Nienaber CA, Myrmel T, Cooper JV, et al. Acute type A aortic dissection in the elderly: clinical characteristics, management, and outcomes in the current era. J Am Coll Cardiol. 2002;40:685-92

45. Debratra M, Eagle KA. Aortic Dissection - An Update. Curr Prob Cardiol. 2005;30:287-325.

46. Besser HW. A hipertensão arterial nas doenças cardiovasculares incapacitantes e "Cardiopatia Grave". Tese de doutorado. Faculdade de Medicina da UFRJ, 2005: 140 p.

\section{Anexos}

\section{Classificação Angiográfica da Insuficiência Mitral de Selle}

Trivial $(1+)$ : contraste entra no átrio esquerdo durante a sístole, sendo eliminado a cada contração

Leve $(2+)$ : opacificação do átrio esquerdo menor que do ventrículo esquerdo, e o contraste não é eliminado com contração atrial

Moderada $(3+)$ : opacificação do átrio esquerdo é igual à do ventrículo esquerdo;

Severa (4+): a presença de um dos 3 achados: opacificação do átrio esquerdo maior que a do ventrículo esquerdo, opacificação do átrio esquerdo no período de uma ejeção sistólica, ou presença de contraste nas veias pulmonares.

Referência: Seller RD, Levy MJ, Amplatz K, Lillehei CW. Retrograde cardioangiography in acquired cardiac disease: technique, indications and interpretation of 100 cases. Am J Cardiol. 1964; 14: 437.

\section{Classificação de Killip no infarto agudo do miocárdio}

Classe I - Sem evidência de insuficiência cardíaca.

Classe II - Achados consistentes com insuficiência cardíaca leve a moderada (B3, estertores crepitantes em menos da metade dos campos pulmonares ou distensão venosa jugilar).

Classe III - Edema pulmonar.

Classe IV - Choque cardiogênico.

Referência: Killip T, 3rd Kimball JT. Treatment of myocardial infarction in a coronary care unit. A two year experience with 250 patients. Am J Cardiol 1967; 20:457.

\section{Classe funcional segundo a New York Heart Association}

Classe I - Sem limitações durante atividade corriqueira

Classe II- Alguma limitação decorrente de dispnéia ou fadiga durante estresse ou exercício moderados.

Classe III - Sintomas com os mínimos esforços que interferem com as atividades diárias.

Classe IV - Inabilidade de realizar qualquer atividade física, dispnéia em repouso.

Referência: The Criteria Committee of the New York Heart Association. Diseases of the Heart and Blood Vessels: Nomenclature and Criteria for Diagnosis. $6^{\text {th }}$ ed. Boston - Mass: Little Brown; 1964.

\section{Escore de angina segundo a Sociedade Canadense}

Classe I - Atividade física habitual, como caminhar ou subir escadas, não provoca angina. Angina ocorre com esforços físicos prolongados e intensos.

Classe II - Discreta limitação para atividades habituais: a angina ocorre ao paciente caminhar ou subir escadas rapidamente, caminhar em aclives, caminhar ou subir escadas após refeições, ou no frio, ao vento, sob estresse emocional, ou apenas durante poucas horas após o despertar. A angina ocorre após caminhar por dois quarteirões planos ou subir mais de um lance de escada em condições normais.

Classe III - Limitação com atividades habituais: a angina ocorre ao caminhar num quarteirão plano ou subir um lance de escada.

Classe IV - Incapacidade de realizar qualquer atividade habitual sem desconforto - os sintomas anginosos podem estar presentes no repouso.

Referência: Campeau L. Grading of angina pectoris [letter]. Circulation. 1976; 54: 522-3. 
Classificação da retinopatia hipertensiva por Keith- Wagener- Barker

Grupo I - Estreitamento arteriolar e alteração leve do reflexo arteriolar.

Grupo II - Estreitamento arteriolar e alteração do reflexo arteriolar mais acentuado e cruzamento arteriolo-venular patológico.
Grupo III - Alteração do grupo II, hemorragia retiniana e exudatos.

Grupo IV - Alteração do grupo III e papiledema.

Referência: Keith NM, Wagener HP, Barker NW. Some different types of essential hypertension: their course and prognosis. Am J Med Sci. 1939; 197:332-43 